\title{
Classification of translation invariant topological Pauli stabilizer codes for prime dimensional qudits on two-dimensional lattices
}

\author{
Jeongwan Haah \\ Microsoft Quantum and Microsoft Research, Redmond, Washington, USA
}

\begin{abstract}
We prove that on any two-dimensional lattice of qudits of a prime dimension, every translation invariant Pauli stabilizer group with local generators and with code distance being the linear system size, is decomposed by a local Clifford circuit of constant depth into a finite number of copies of the toric code stabilizer group (abelian discrete gauge theory). This means that under local Clifford circuits the number of toric code copies is the complete invariant of topological Pauli stabilizer codes. Previously, the same conclusion was obtained under the assumption of nonchirality for qubit codes or the Calderbank-Shor-Steane structure for prime qudit codes; we do not assume any of these.
\end{abstract}

\section{INTRODUCTION AND RESULT}

Topological phases of matter of gapped systems refer to equivalence classes of physical systems where two systems are deemed equivalent if they can be smoothly deformed to each other while preserving the energy gap above the ground state subspace. By definition, any characteristic of a topological phase of matter should be robust under any local perturbations. The study of topological phases of matter thus focuses, to a large extent, on identifying invariants of systems under smooth deformations and on concrete models that exhibit different invariants. Indeed, many sophisticated model Hamilonians are constructed to date in various dimensions in the absence or presence of symmetries [1-3]. However, the classification (i.e., finding complete invariants) of topological phases remains still an active problem.

Here we present a classification theorem on translation-invariant topological Pauli stabilizer code Hamiltonians in two spatial dimensions. These are a class of Pauli stabilizer codes [4-7] whose stabilizer group generators define a local Hamiltonian that exhibits intrinsic topological order [8, 9]. By construction, the Hamiltonian terms are commuting, and all operators that determine the topological invariants are tensor products of Pauli matrices. Simply put, our result is that there is only the toric code (abelian discrete gauge theory) in two dimensions.

Though Pauli stabilizer models are simple to calculate about, some Hamiltonians in this class realize exotic phases of matter (e.g. fracton topological order [10]) in three or higher spatial dimensions. This makes our conclusion in two dimensions more interesting; later we will highlight 
the steps in the proof of our result where it is important to work in two dimensions.

Before we present our rigorous statement, let us review previous reasoning that has alluded our result. Believing in the effective description by unitary modular tensor categories (UMTC) [11, 12], we can tabulate possible topological phases of matter realized by two-dimensional topological Pauli stabilizer codes. Indeed, if we consider generalized Pauli operators (or discrete Weyl operators) over a system of qudits of dimension $p$, which we will define below, the topological spins for a Pauli stabilizer code model must be valued in $p$-th roots of unity and the UMTC is determined by a quadratic form $\theta$ over $\mathbb{Z} / p \mathbb{Z}$; see e.g. $[13, \S 5]$ and references therein. When $p$ is a prime (so that the topological spins are valued in a finite field $\mathbb{F}_{p}$ ) nondegenerate quadratic forms are particularly simple $[14,15]$. If we ignore direct summands of hyperbolic forms, which correspond to the toric code phase [9], then the only nontrivial possibilities are listed as follows. Let $v$ denote a general vector in the domain $V$ (an $\mathbb{F}_{p}$-vector space) of a quadratic form $\theta: V \rightarrow \mathbb{F}_{p}$. Recall that an $n$-dimensional quadratic form over $\mathbb{F}_{p}$ is a homogeneous polynomial in $n$ variables of total degree 2 with coefficients in $\mathbb{F}_{p}$.

1. $(p=2)$ a two-dimensional form $\theta_{3 F}(v)=v_{1}^{2}+v_{1} v_{2}+v_{2}^{2}$ which corresponds to the "threefermion" theory.

2. $\left(p \equiv 3 \bmod 4\right.$ so the Witt group is $\left.\mathbb{Z}_{4}\right)$ a one-dimensional form $\theta_{1}(v)=v^{2}$, its time-reversal conjugate $-\theta_{1}$, or a direct $\operatorname{sum} \theta_{1} \oplus \theta_{1} \cong\left(-\theta_{1}\right) \oplus\left(-\theta_{1}\right)$.

3. $\left(p \equiv 1 \bmod 4\right.$ so the Witt group is $\left.\mathbb{Z}_{2} \oplus \mathbb{Z}_{2}\right)$ a one-dimensional form $\theta_{1}(v)=v^{2}$, another one-dimensional form $\theta_{\alpha}(v)=\alpha v^{2}$ where $\alpha$ is any nonsquare element of $\mathbb{F}_{p}$, or their direct $\operatorname{sum} \theta_{1} \oplus \theta_{\alpha}$.

This motivates us to ask whether these candidates are exhaustive and whether every candidate can be realized in a lattice model. For Pauli stabilizer code models the problem becomes a question on locally generated abelian multiplicative groups of Pauli operators.

Previously, Bombín [16] has studied topological stabilizer groups in two dimensions that are translation invariant on systems of qubits $(p=2)$ and concluded that, up to locality preserving automorphisms of operator algebra ${ }^{1}$ that map every Pauli matrix to a product of Pauli matrices $[18$, 19], any such group is a direct sum of copies of the toric code stabilizer group and a trivial stabilizer group for a product state if the topological charge content is nonchiral, i.e., its decomposition does

\footnotetext{
${ }^{1}$ Locality preserving automorphisms (also called quantum cellular automata) are not necessarily shallow quantum circuits. It is only recent results $[17,18]$ that such a locality preserving automorphisms in two dimensions is actually a shallow quantum circuit.
} 
not contain the three-fermion theory. Any chiral theory in two dimensions is widely believed to be not realizable by any commuting Hamiltonian, but there are at least two definitions of chirality in this statement: one from thermal energy current [12, App.D] and the other from algebraic theory of anyons [20, cf.Prop.6.20]. We are unaware of formal connections between these notions.

In relation to [16], the present author has studied [21] a similar translation invariant case with qudits of a prime dimension under the assumption that the group be generated by tensor products of $X$ and tensor products of $Z$ (the Calderbank-Shor-Steane structure [4,5]) and concluded that every such stabilizer group is a direct sum of those for the toric code after a geometrically local circuit of control-Not gates.

Recently a new ingredient was obtained [22], which proves that every translation invariant topological Pauli stabilizer code on prime qudits must have a nontrivial "boson." A boson is by definition an excitation with topological spin $1 \in \mathbb{C}$ (or $0 \in \mathbb{Z} / p \mathbb{Z}$ in our setting). When topological spin corresponds to a quadratic form, the existence of a boson means that the quadratic form $\theta$ has a nonzero vector whose value is zero, in which case the quadratic form is called isotropic. Hence, it rules out all the nontrivial possibilities listed above where we have listed all anisotropic quadratic forms over prime finite fields. This has left a question of whether the equivalence of quadratic forms of topological spins implies the equivalence of the Pauli stabilizer groups under Clifford circuits; the latter is stronger than just the equivalence of the corresponding topological phases of matter. In this paper we answer this question in the affirmative, closing the classification problem of two-dimensional topological Pauli stabilizer codes over prime dimensional qudits up to Clifford circuits in the translation invariant case. Let us state our result precisely.

We first recall standard definitions. A generalized Pauli matrix (discrete Weyl operators) for a $p$-dimensional qudit $\mathbb{C}^{p}$ is any product of

$$
\exp \left(\frac{2 \pi \mathrm{i}}{p}\right) I, \quad X=\sum_{j \in \mathbb{Z}_{p}}|j+1\rangle\langle j| \quad \text { and } \quad Z=\sum_{j \in \mathbb{Z}_{p}} \exp \left(\frac{2 \pi \mathrm{i}}{p} j\right)|j\rangle\langle j| .
$$

These are defined for any integer $p \geq 1$, but in this paper $p$ is always a prime. A generalized Pauli operator is any finite tensor product of generalized Pauli matrices. A Clifford gate is a finitely supported unitary that maps every generalized Pauli operator to a generalized Pauli operator, and a Clifford circuit is a finite composition of layers of nonoverlapping Clifford gates each of which is supported on a ball of a uniformly bounded radius. The number of layers in a circuit is the depth of the circuit. A trivial stabilizer group is the group of generalized Pauli operators generated by $Z$ on every qudit. The toric code stabilizer group [9] is an abelian group of generalized Pauli operators on a two-dimensional lattice $\mathbb{Z}^{2}$ with two $p$-dimensional qudits per lattice point, 
generated by $X_{s+\hat{y}, 1} X_{s, 1}^{\dagger} X_{s+\hat{x}, 2}^{\dagger} X_{s, 2}$ and $Z_{s-\hat{x}, 1}^{\dagger} Z_{s, 1} Z_{s-\hat{y}, 2}^{\dagger} Z_{s, 2}$ for all sites $s$.

Theorem I.1. Let $\mathcal{S}$ be an abelian group of generalized Pauli operators acting on a two-dimensional square lattice $\mathbb{Z}^{2}$ with $q \geq 1$ qudits of a prime dimension $p$ per lattice point. Suppose that

1. (No frustration) if $\omega I \in \mathcal{S}$ for $\omega \in \mathbb{C}$, then $\omega=1$,

2. (Translation invariance up to a phase) if $P \in \mathcal{S}$, then for every translate $P^{\prime}$ of $P$, we have $\omega P^{\prime} \in \mathcal{S}$ for some $p$-th root of unity $\omega \in \mathbb{C}$, and

3. (Topological order) if a generalized Pauli operator $P$ commutes with every operator of $\mathcal{S}$, then $\omega P \in \mathcal{S}$ for some $\omega \in \mathbb{C}$.

Then, there exists a Clifford circuit of finite depth that maps $\mathcal{S}$ into a direct sum $\mathcal{T}^{\oplus n} \oplus \mathcal{Z}$ for some $n \geq 0$ where $\mathcal{T}$ is the toric code stabilizer group, and $\mathcal{Z}$ is the trivial stabilizer group. Here the circuit is translation invariant with respect to a subgroup of the full translation group $\mathbb{Z}^{2}$ with finite index.

Physically, the stabilizer group as a whole is more important than a generating set since any local generating set can be used to define a gapped Hamiltonian but the quantum phase of matter only depends on the group $[23, \S 2]$. This is why we have stated our theorem in terms of groups. Our result should be understood as the scope of the topological phases that can be realized by unfrustrated commuting Pauli Hamiltonians.

One might wonder why there is no reference to the length scale of the topological order. This is because the definition of topological order in [24] is for a family of finite lattices, whereas we work with infinite lattices. Our assumption implies the topological order condition of [24] with length scale being the linear system size under periodic boundary conditions [23]. Any implicit finite number in the theorem such as the depth of the Clifford circuit and the index of the translation subgroup gives a constant uniform bound on a corresponding quantity for an infinite family of finite systems.

Note that our theorem assumes a finite dimensional degrees of freedom per site. This is not just a technical convenience but rather a fundamentally important assumption. Indeed, in a limit $p \rightarrow \infty$ that would produce a rotor $U(1)$ model, the basic result that any excitation is attached to a string operator and hence is mobile, cease to be true in general; consider 2+1D analogues of [25]. Moreover, when it comes to rotor models we do not have any stability result against perturbations such as [24] but only an instability result [26]. 
It remains an open problem to relax the translation invariance. One might be able to promote an arbitrary system to a periodic system [27], but it appears difficult to adapt the present classification proof directly to nonperiodic situations; the argument for the existence of a boson in [22] relies on bilinear forms over a field of fractions in one variable, for which the translation invariance is used.

Even assuming translation invariance, there is a quantitative question left. In our mapping from a given stabilizer group to a direct sum of toric code stabilizer groups, we had to break the translation invariance down to a smaller group. To some extent this is necessary; a translation group may act nontrivially on the fusion group of anyons [28], while for the toric code the action is trivial. However, almostly surely, our choice of smaller translation group is not the biggest possible; in fact, we will not keep track of the index of this translation subgroup. One can then ask what the precise order (exponent) of the translation group action on the fusion group of anyons is. Generally this question should be answered as a function of interaction range and unit cell size. A lower bound that is exponential in the interaction range is known [23, §7 Rem. 3], but the upper bound is largely open.

The rest of this paper constitutes the proof of Theorem I.1.

\section{TRANSCRIPTION TO POLYNOMIALS}

Following [23] (see also [21] and a summary section [22, §IV.A]) we transcribe the problem into a polynomial framework by regarding translation invariant groups of generalized Pauli operators modulo phase factors, which are abelian, as modules over the translation group algebra

$$
R=\mathbb{F}_{p}\left[x^{ \pm}, y^{ \pm}\right]
$$

In particular, the abelianized group of generalized Pauli operators is a free module $R^{2 q}$ where $q$ is the number of qudits per lattice site (unit cell), equipped with a nondegenerate symplectic form that captures commutation relations. Below we will not distinguish generalized Pauli operators from an element of $R^{2 q}$ as the phase factors will not be important.

Already this perspective implies the following: Note that in Theorem I.1 we do not assume that the stabilizer group is generated by operators whose supports are contained in disks of a uniformly bounded radius; that is, we do not assume that the stabilizer group has a local generating set. However, the supposition of Theorem I.1 implies that the stabilizer group has a local generating set. The group of all generalized Pauli operators (each of which is finitely supported) up to phase factors is $R^{2 q}$, which is a finitely generated module over $R$. The stabilizer group modulo phase 
factors is a subset of $R^{2 q}$ but the translation invariance implies that this subset is actually a submodule. Since $R$ is Noetherian, the stabilizer submodule must be finitely generated, which means that the stabilizer group does have local generators with uniformly bounded support size, where the bound is given by a finite generating set for the stabilizer submodule.

We use $\mathbb{F}_{p}$-linear ring homomorphisms $\phi^{(m)}: \mathbb{F}_{p}\left[x^{\prime \pm}, y^{\prime \pm}\right] \rightarrow R$ such that $x^{\prime} \mapsto x^{m}$ and $y^{\prime} \mapsto y^{m}$ indexed by positive integer $m$ to denote coarse-graining, which induces formally a covariant functor $\phi_{\#}^{(n)}$ on the category of $R$-modules. The domain of this morphism is interpreted as the group algebra for a smaller translation group, enlarging the unit cell of the qudit system $m \times m$ times as large as the original one. We use $\cdots$ to denote the $\mathbb{F}_{p}$-linear involution of $R$ such that $x \mapsto \bar{x}=x^{-1}$ and $y \mapsto \bar{y}=y^{-1}$, and $\dagger$ the involution followed by transpose for matrices over $R$. Let $I_{q}$ denote the $q \times q$ identity matrix. We fix specific matrices over $R$ :

$$
\lambda_{q}=\left(\begin{array}{cc}
0 & I_{q} \\
-I_{q} & 0
\end{array}\right), \quad \epsilon_{0}=\left(\begin{array}{cccc}
x-1 & y-1 & 0 & 0 \\
0 & 0 & \bar{y}-1 & -\bar{x}+1
\end{array}\right) \quad \text { and } \quad \sigma_{0}=\left(\epsilon_{0} \lambda_{q}^{-1}\right)^{\dagger}
$$

where $\epsilon_{0}$ describes the $\mathbb{Z}_{p}$ toric code [9] on the square lattice [23, §5 Ex. 2]. For the clarity in notation we define a matrix $E_{i, j}(a)$ for any $a \in R$ as

$$
\left[E_{i, j}(a)\right]_{\mu \nu}=\delta_{\mu \nu}+\delta_{\mu i} \delta_{\nu j} a \quad \text { where } \delta \text { is the Kronecker delta. }
$$

Definition II.1. For a given positive integer $q$, the following $2 q \times 2 q$ matrices generate the elementary symplectic group denoted by $\operatorname{ESp}^{\dagger}(q ; R)$. Below $a \in R^{\times}$is any monomial.

$$
\begin{array}{clr}
\text { Hadamard: } & E_{i, i+q}(-1) E_{i+q, i}(1) E_{i, i+q}(-1) & \text { where } 1 \leq i \leq q, \\
\text { control-Phase: } & E_{i+q, j}(a) E_{j+q, i}(\bar{a}) & \text { where } 1 \leq i, j \leq q, \\
\text { control-Not: } & E_{i, j}(a) E_{j+q, i+q}(-\bar{a}) & \text { where } 1 \leq i \neq j \leq q, \\
\text { extra gate: } & E_{i, i}(a-1) E_{i+q, i+q}\left(a^{-1}-1\right) & \text { where } 1 \leq i \leq q .
\end{array}
$$

The symplectic group denoted by $\operatorname{Sp}^{\dagger}(q ; R)$ consists of all $U \in \operatorname{Mat}(2 q ; R)$ such that $U^{\dagger} \lambda_{q} U=$ $\lambda_{q} \cdot^{2}$

Note that the control-Phase ${ }^{3}$ gate with $i=j$ is equivalent to $E_{i+q, i}(f)$ for some $f=\bar{f}$, and conversely any $E_{i+q, i}(f)$ with $f=\bar{f}$ can be written as the control-Phase with $i=j$ since any such $f$ is of form $a+\bar{a}$ for some nonunique $a$. The extra gate is the identity if $p=2$.

\footnotetext{
${ }^{2}$ The $\dagger$ in the notation (E)Sp ${ }^{\dagger}$ refers to the fact that we collect matrices $U$ that obeys $U^{\dagger} \lambda_{q} U=\lambda_{q}$. Sometimes a different group consisting of $U$ such that $U^{T} \lambda_{q} U=\lambda_{q}$ is considered in literature, and our notation differentiate them.

${ }^{3}$ This includes the induced action by the "phase" gate $\operatorname{diag}(1, \mathrm{i})$ but not $\operatorname{diag}(1,1,1, \mathrm{i})$ for qubits $(p=2)$. Hence, this terminology may be inconsistent with that elsewhere.
} 
The elementary symplectic group is a subgroup of the symplectic group as one can verify, but they are not equal if $R$ was a Laurent polynomial ring of three or more variables [18]. If $R=$ $\mathbb{F}_{p}\left[x^{ \pm}, y^{ \pm}\right]$, the Laurent polynomial ring of two variables, then for every $U \in \operatorname{Sp}^{\dagger}(q ; R)$ there exists a positive integer $m$ such that $\phi_{\#}^{(m)}(U) \in \mathrm{ESp}^{\dagger}\left(m^{2} q ; R\right)[18]$. That is, every symplectic transformation on two-dimensional lattice is a Clifford circuit with weaker translation invariance. Hence, in the present paper where we only have two-dimensional lattices, we do not have to distinguish $\mathrm{ESp}^{\dagger}$ and $S \mathrm{p}^{\dagger}$.

Theorem II.2 (Implying Theorem I.1). Let $\sigma$ be a $2 q \times t$ matrix over $R$, interpreted as a map acting on the left of a column vector of length $t$, such that

$$
\operatorname{ker} \sigma^{\dagger} \lambda_{q}=\operatorname{im} \sigma
$$

Then, there exist an integer $m \geq 1$ and a matrix $E \in \mathrm{ESp}^{\dagger}\left(m^{2} q ; R\right)$ such that

$$
E \operatorname{im} \phi_{\#}^{(m)}(\sigma)=\left(\operatorname{im}\left(\begin{array}{c}
I_{m^{2} q-k} \\
0
\end{array}\right)\right) \oplus \bigoplus^{k / 2} \operatorname{im} \sigma_{0}
$$

where the images are over $R^{\prime}=\mathbb{F}_{p}\left[x^{ \pm m}, y^{ \pm m}\right] \subseteq R$ and $k$ is an even integer that is equal to the $\mathbb{F}_{p}$-dimension of the torsion submodule of coker $\sigma^{\dagger}$.

Proof of the transcription. Any translation invariant abelian group of generalized Pauli operators corresponds to a submodule $S$ of the $R$-module $P$ of all generalized Pauli operators (forgetting phase factors) with the property that $v^{\dagger} \lambda_{q} v=0$ for any $v \in S$ [23, Prop. 1.2]. Picking a generating set for the module $S$, which amounts to writing $S=\operatorname{im} \sigma$ for some matrix $\sigma$ over $R$, we have a matrix equation $\sigma^{\dagger} \lambda_{q} \sigma=0$. The topological order condition [24] for unfrustrated commuting Pauli Hamiltonians is equivalent to $\operatorname{ker} \sigma^{\dagger} \lambda_{q}=\operatorname{im} \sigma$ [23, Lem. 3.1]. An elementary symplectic trasformation is always induced by a Clifford circuit of finite depth [23, §2.1]. These symplectic transformations are forgetful only of a conjugation by a (possibly infinitely supported) generalized Pauli operator [23, Prop. 2.2]. The module $\mathrm{im} \sigma_{0}$ is the same as the toric code stabilizer group.

\section{PROOF}

The proof of Theorem II.2 is by induction in the vector space dimension $k$ of the torsion part of coker $\epsilon$ where $\epsilon=\sigma^{\dagger} \lambda_{q}$ is the excitation map of the given stabilizer group. It has been proved that the excitation map $\epsilon$ can be chosen (as it depends on a generating set for the stabilizer group) such that coker $\epsilon$ is a torsion module if $R$ is the Laurent polynomial ring with two variables, and 

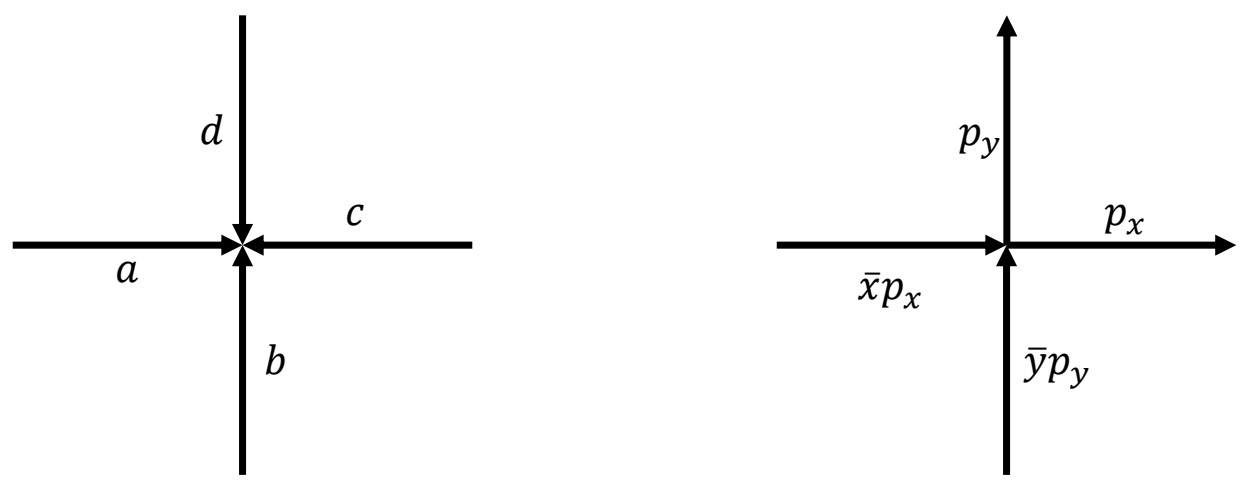

FIG. 1. Left: Movers (hopping operators) that send a topological charge $e$ to the center. Commutation relations of any triple of the movers determine the topological spin $\theta$ of $e$; Lemma III.2. Right: For a boson $(\theta=0)$ we can choose movers $p_{x}, p_{y}$ on a coarse scale such that they always commute; Lemma III.4.

furthermore after a suitable choice of a smaller translation group (coarse-graining) the annihilator of coker $\epsilon$ becomes a maximal ideal $\mathfrak{m}=(x-1, y-1) \subset R[23, \S 7$ Thm. 4]. Such $\epsilon$ must be $q \times 2 q$ to satisfy the topological order condition $\operatorname{ker} \epsilon=\operatorname{im} \lambda_{q} \epsilon^{\dagger}$. Hence, without loss of generality we begin with an extra assumption, whenever coker $\epsilon \neq 0$, that

$$
\text { ann coker } \epsilon=\mathfrak{m}=(x-1, y-1) \text {. }
$$

This implies that $k$ is finite. We will extract a copy of the toric code whenever $k \geq 2$, decreasing this dimension $k$ by 2 . This is done by trivializing string operators that transports bosons whose existence is proved in [22, Cor. III.20]. The possibility $k=1$ will be ruled out in the course of the proof. The case $k=0$ is treated in $[22, \S I V . B]$ where a Clifford quantum cellular automaton is constructed to map the stabilizer group to the trivial stabilizer group. But it is shown that any Clifford QCA in two dimensions is in fact a Clifford circuit with weaker translation invariance up to a shift [18]. A shift QCA does not change the stabilizer group at all. These will complete the proof.

\section{A. Topological spin}

We consider the topological spins of the excitations of a given topological stabilizer group. We will define them using hopping operators for the excitations in a way that is tailored to our setting. Recall that two generalized Pauli operators represented as two column vectors $u, v$ over $R$, commute 
with each other if and only if

$$
[u, v]=-[v, u] \in \mathbb{F}_{p}
$$

vanishes, where $[u, v]$ is the coefficient of $x^{0} y^{0}=1 \in R$ in $u^{\dagger} \lambda_{q} v$ [23, Prop.1.2]. Let us introduce a notation for any $a, b, c \in R^{2 q}$

$$
[a, b, c]=[a, b]+[b, c]+[c, a]
$$

It is obvious that $[a, b, c]=[b, c, a]=[c, a, b]$.

Definition III.1. Assuming Eq. (7), an excitation is any element in the codomain of $\epsilon$ or equivalently any element in the domain of $\sigma$. An excitation $e$ is nontrivial if $e \notin \operatorname{im} \epsilon$. A (topological) charge is then an equivalence class of excitations modulo trivial ones. An $x$-mover $p_{x}(e)$ for an excitation $e$ is any generalized Pauli operator $p \in R^{2 q}$ such that $\epsilon(p)=(x-1) e$. Likewise, a $y$-mover $p_{y}(e)$ is any $p \in R^{2 q}$ such that $\epsilon(p)=(y-1) e$. The topological spin $\theta(e) \in \mathbb{F}_{p}$ of an excitation is

$$
\begin{aligned}
\theta(e) & =\lim _{n \rightarrow+\infty}\left[a_{n}, b_{n}, c_{n}\right] \\
\text { where } \quad a_{n} & =\left(x^{-n}+x^{-n+1}+\cdots+x^{-1}\right) p_{x}(e), \\
b_{n} & =\left(y^{-n}+y^{-n+1}+\cdots+y^{-1}\right) p_{y}(e), \quad \text { and } \\
c_{n} & =-\left(1+x+x^{2}+\cdots+x^{n-1}\right) p_{x}(e) .
\end{aligned}
$$

The topological spin can and should be defined much more generally, but we use this narrow definition which is sufficient for this paper. The existence of movers for any excitation is precisely the content of Eq. (7). Note that movers are not unique since any element of $\operatorname{ker} \epsilon=\operatorname{im} \sigma$ can be added to them. Our definition of topological spin is due to [29] using commutation relations among three hopping operators (movers) attached to an excitation; we have transcribed it into our additive notation. The limit as $n \rightarrow \infty$ exists because $a_{n}, b_{n}, c_{n}$ are long "string" operators and string segments far away from the origin of the lattice always commute and hence the sequence in $n$ becomes constant eventually. Pictorially, $a_{n}$ is inserting $e$ from the left infinity to the origin, $b_{n}$ from the bottom, and $c_{n}$ from the right. See Fig. 1 .

Lemma III.2. First, $\theta(e)$ is independent of the choices of the movers. Second, $\theta\left(e^{\prime}\right)=\theta(e)$ if $e^{\prime}-e$ is trivial. Third, $\theta(e)=\theta\left(\phi_{\#}^{(m)}(e)\right)$ for any positive integer $m$. Fourth, $\theta(e)=\left[a_{n}, b_{n}, d_{n}\right]=$ $\left[a_{n}, c_{n}, d_{n}\right]=\left[b_{n}, c_{n}, d_{n}\right]$ as $n \rightarrow \infty$ where

$$
d_{n}=-\left(1+y+y^{2}+\cdots+y^{n-1}\right) p_{y}(e)
$$


The fourth claim is basically that the triple commutator can consist of any string operators as long as they circle around the excitation $e$ counterclockwise.

Proof. First, the movers are unique up to $\operatorname{ker} \epsilon=\operatorname{im} \sigma$, and hence $\tilde{p}_{x, y}(e)=p_{x, y}(e)+s_{x, y}$ for some $s_{x}, s_{y} \in \operatorname{im} \sigma$ is the most general form of movers. Let us examine

$$
\tilde{\theta}(e)-\theta(e)=\lim _{n \rightarrow \infty}\left[\tilde{a}_{n}-a_{n}, b_{n}-\tilde{c}_{n}\right]+\left[\tilde{b}_{n}-b_{n}, c_{n}-\tilde{a}_{n}\right]+\left[\tilde{c}_{n}-c_{n}, a_{n}-\tilde{b}_{n}\right]
$$

where those with tilde are by the modified movers $\tilde{p}_{x, y}(e)$, and show that each term vanishes on its own. The first term is $\left[\left(x^{-n}+x^{-n+1}+\cdots+x^{-1}\right) s, b_{n}-\tilde{c}_{n}\right]$ for some sufficiently large $n$. The difference $b_{n}-\tilde{c}_{n}$ creates excitations near $(0,-n) \in \mathbb{Z}^{2}$ and $(n, 0) \in \mathbb{Z}^{2}$ where $\left(x^{-n}+x^{-n+1}+\cdots+x^{-1}\right) s$ does not have any support, and hence the commutator vanishes. The other two terms vanish for completely parallel reasons.

Second, let $g \in R^{2 q}$ be a finitely supported operator such that $\epsilon(g)=e^{\prime}-e$. By the first claim we can choose movers for $e^{\prime}$ as $p_{x}\left(e^{\prime}\right)=p_{x}(e)+(x-1) g$ and $p_{y}\left(e^{\prime}\right)=p_{y}(e)+(y-1) g$. Then, $a_{n}\left(e^{\prime}\right)-a_{n}(e)=\left(1-x^{-n}\right) g, b_{n}\left(e^{\prime}\right)-b_{n}(e)=\left(1-y^{-n}\right) g$, and $c_{n}\left(e^{\prime}\right)-c_{n}(e)=\left(1-x^{n+1}\right) g$. Now we see

$$
\begin{aligned}
\theta\left(e^{\prime}\right)-\theta(e)= & {\left[g-x^{-n} g, b_{n}\right]+\left[a_{n}, g-y^{-n} g\right]+\left[g-x^{-n} g, g-y^{-n} g\right] } \\
& +\left[g-y^{-n} g, c_{n}\right]+\left[b_{n}, g-x^{n+1} g\right]+\left[g-y^{-n} g, g-x^{n+1} g\right] \\
& +\left[g-x^{n+1} g, a_{n}\right]+\left[c_{n}, g-x^{-n} g\right]+\left[g-x^{n+1} g, g-x^{-n} g\right] \\
= & {\left[g, b_{n}\right]+\left[a_{n}, g\right]+[g, g] } \\
& +\left[g, c_{n}\right]+\left[b_{n}, g\right]+[g, g] \\
& +\left[g, a_{n}\right]+\left[c_{n}, g\right]+[g, g] \\
= & 0
\end{aligned}
$$

where the first equality is by linearity of $[\cdot, \cdot]$, the second equality is because operators of disjoint support commute (e.g. $\left[x^{-n} g, b_{n}\right]=0$ for all sufficiently large $n$ ), and the third equality is because $[g, g]=0$ and $[u, v]=-[v, u]$ for any $u, v \in R^{2 q}$.

Third, if we have movers $p_{x}, p_{y}$ for $e$, we can choose movers $p_{x^{\prime}}, p_{y^{\prime}}$ for $\phi_{\#}^{(m)}(e)$ as $p_{x^{\prime}}=\phi_{\#}^{(m)}((1+$ $\left.\left.x+\cdots+x^{m-1}\right) p_{x}\right)$ and $p_{y^{\prime}}=\phi_{\#}^{(m)}\left(\left(1+y+\cdots+y^{m-1}\right) p_{y}\right)$. The commutator is oblivious to $\phi_{\#}^{(m)}$ and the new movers are just longer (larger $n$ ) string operators.

Fourth, we examine the difference, omitting the subscript $n$ for brevity:

$$
\begin{aligned}
{[a, b, c]-[a, b, d] } & =[a, b]+[b, c]+[c, a]-[a, b]-[b, d]-[d, a] \\
& =[b-a, c-d] .
\end{aligned}
$$


The operator $b-a$ creates excitations at $(0,-n),(-n, 0) \in \mathbb{Z}^{2}$ while $c-d$ is supported around the positive $x$ and $y$ axes. Hence, we can find a straight diagonal string operator $f$ connecting $(0,-n)$ and $(-n, 0)$ such that $[f, c-d]=0$ (for their disjoint support) and $\epsilon(b-a+f)=0$. Since $\operatorname{ker} \epsilon=\operatorname{im} \sigma$, we know $b-a+f \in \operatorname{im} \sigma$. Similarly, we can find a straight diagonal string operator $h$ connecting $(n, 0) \in \mathbb{Z}^{2}$ and $(0, n) \in \mathbb{Z}^{2}$ such that $c-d+h \in \operatorname{ker} \epsilon=\operatorname{im} \sigma$ and $[b-a+f, h]=0$. This means that $[b-a, c-d]=[b-a+f, c-d+h]$, but the latter is zero since $[u, v]=0$ for any $u, v \in \operatorname{im} \sigma$. This proves that $\theta(e)=\lim _{n \rightarrow \infty}\left[a_{n}, b_{n}, c_{n}\right]=\lim _{n \rightarrow \infty}\left[a_{n}, b_{n}, d_{n}\right]$. Similarly,

$$
\begin{aligned}
{[a, b, d]-[a, c, d] } & =[a, b]+[b, d]+[d, a]-[a, c]-[c, d]-[d, a] \\
& =[a-d, b-c]
\end{aligned}
$$

The operator $a-d$ creates excitations at $(-n, 0),(0, n) \in \mathbb{Z}^{2}$ and the operator $b-c$ creates excitations at $(0,-n),(n, 0) \in \mathbb{Z}^{2}$, which can be turned into elements of im $\sigma$ by "capping off" by straight diagonal string operators and the commutator $[a-d, b-c]$ is shown to vanish. A parallel argument to shows that $\left[a_{n}, c_{n}, d_{n}\right]=\left[b_{n}, c_{n}, d_{n}\right]$ for all sufficiently large $n$.

Therefore, $\theta$ is well defined on the set of all topological charges (equivalence classes of excitations). In fact, $\theta$ is a quadratic form on the $\mathbb{F}_{p}$-vector space coker $\epsilon{ }^{4}$ If $\theta(e)=0$, we call $e$ a boson.

Lemma III.3 (Cor. III.20 of [22]). If coker $\epsilon \neq 0$, then there exists a nontrivial excitation $e \notin \operatorname{im} \epsilon$ such that $\theta(e)=0$, i.e., a nontrivial boson exists.

\section{B. Simplification of movers and excitation maps}

The rest of the proof of Theorem II.2 consists of elementary - a little complicated but not difficult - computation. In Lemma III.4 below we will choose movers for a nontrivial boson such that they commute with any of its translates. In Lemma III.6 we will further simplify the movers to determine two columns of $\epsilon$. Then, using the exactness condition Eq. (5), we will find an accompanying row of $\epsilon$; this will rule out the possibility $k:=\operatorname{dim}_{\mathbb{F}_{p}}$ coker $\epsilon=1$. The determined columns and row will be further simplified in Lemma III.7 and in turn will single out a direct summand $\epsilon_{0}$ from $\epsilon$.

\footnotetext{
4 The definition of a quadratic form [15] requires that its polar form $S\left(e, e^{\prime}\right)=\theta\left(e+e^{\prime}\right)-\theta(e)-\theta\left(e^{\prime}\right)$ be bilinear over $\mathbb{F}_{p}$. This is true, and in fact one can show that this modular $S$ is precisely the commutation relation of all logical operators of the finite dimensional stabilizer code on any sufficiently large but finite 2-torus. In particular, $S$ is nondegenerate. One can classify the quadratic forms $\theta$ based only on the fact that it is $\mathbb{F}_{p}$-valued and $S$ is nondegenerate, and the result is in Introduction.
} 
Lemma III.4. Under a choice of a sufficiently large unit cell (reducing the translation group to a subgroup of a finite index via $\phi_{\#}^{(m)}$ for some $\left.m\right)$, the movers $p_{x^{\prime}}(e), p_{y^{\prime}}(e)$ for a boson e can be chosen such that $p_{x^{\prime}}(e)^{\dagger} \lambda_{m^{2} q} p_{y^{\prime}}(e)=p_{x^{\prime}}(e)^{\dagger} \lambda_{m^{2} q} p_{x^{\prime}}(e)=p_{y^{\prime}}(e)^{\dagger} \lambda_{m^{2} q} p_{y^{\prime}}(e)=0$. Furthermore, the entries of $p_{x^{\prime}}(e)$ as a Laurent polynomial vector over $R^{\prime}=\mathbb{F}_{p}\left[x^{\prime \pm}, y^{\prime \pm}\right]$ are $\mathbb{F}_{p}$-linear combinations

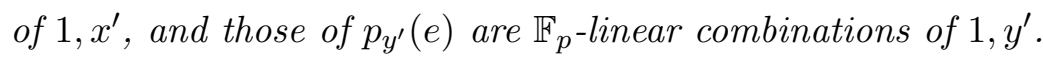

That is, the movers and all their translates can be made commuting.

Proof. Let us drop the reference to $e$ since we fix $e$. Choose arbitrary movers $p_{x}, p_{y}$ with respect to $R ; \epsilon\left(p_{x}\right)=(x-1) e$ and $\epsilon\left(p_{y}\right)=(y-1) e$. If $R^{\prime}=\mathbb{F}_{p}\left[x^{\prime \pm}, y^{\prime \pm}\right]$ injects into $R$ by $\phi^{(m)}: x^{\prime} \mapsto$ $x^{m}, y^{\prime} \mapsto y^{m}$, then a mover $p_{x^{\prime}}$ with respect to $R^{\prime}$, i.e., $\left(x^{\prime}-1\right) e=\left(\phi_{\#}^{(m)} \epsilon\right)\left(p_{x^{\prime}}\right)$, can be chosen as

$$
p_{x^{\prime}}=s_{\text {tail }}+\left(1+x+\cdots+x^{m-1}\right) p_{x}+s_{\text {head }}
$$

where we did not write $\phi_{\#}^{(m)}$ for brevity. The mover $p_{x^{\prime}}$ (at a coarser lattice) is the $m$ movers $p_{x}$ aligned along the moving direction with its "head" near $(m, 0) \in \mathbb{Z}^{2}$ and "tail" near the origin modified by $s_{\text {head,tail }} \in \operatorname{im} \sigma$. Similarly,

$$
p_{y^{\prime}}=t_{\text {tail }}+\left(1+y+\cdots+y^{m-1}\right) p_{y}
$$

with $t_{\text {tail }} \in \operatorname{im} \sigma$ near the origin. (Note that $t_{\text {head }}$ is absent.) We wish to choose these head and tail modifiers such that

$$
\begin{array}{ll}
{\left[\overline{x^{\prime}} p_{x^{\prime}}, \overline{y^{\prime}} p_{y^{\prime}}\right]=0} & \text { by some } s_{\text {head }}, \\
{\left[\overline{y^{\prime}} p_{y^{\prime}},-p_{x^{\prime}}\right]=0} & \text { by some } s_{\text {tail }}, \\
{\left[-p_{y^{\prime}}, \overline{x^{\prime}} p_{x^{\prime}}\right]=0} & \text { by some } t_{\text {tail }} .
\end{array}
$$

These are equations over $\mathbb{F}_{p}$ with unknowns $s_{\text {head }}, s_{\text {tail }}, t_{\text {tail }}$ and can be solved by inspecting one by one in order. The first equation contains neither $s_{\text {tail }}$ nor $t_{\text {tail }}$ as they are far away from the origin at which $\overline{x^{\prime}} p_{x^{\prime}}$ and $\overline{y^{\prime}} p_{y^{\prime}}$ may intersect. Likewise, the second equation contains neither $s_{\text {head }}$ nor $t_{\text {tail }}$. Since a mover must have a nonzero commutator with some element of im $\sigma$ near its head and tail, we conclude that there exist $s_{\text {head }}$ and $s_{\text {tail }}$ satisfying the first two equations. The third equation contains the two unknowns $t_{\text {tail }}$ and $s_{\text {head }}$, but given $s_{\text {head }}$ determined we can certainly solve it for $t_{\text {tail }}$. For a large enough $m$, the movers $p_{x^{\prime}}$ and $p_{y^{\prime}}$ are long string operators, and the choices of head and tail modifiers are independent of all sufficiently large $m$. 
The sequence of Eq. (10) in $n$ constructed by $p_{x^{\prime}}$ and $p_{y^{\prime}}$ is already constant starting from $n=1$. Since $e$ is a boson, the fourth claim of Lemma III.2 implies that

$$
\begin{aligned}
& 0=\left[\overline{x^{\prime}} p_{x^{\prime}}, \overline{y^{\prime}} p_{y^{\prime}}\right]+\left[\overline{y^{\prime}} p_{y^{\prime}},-p_{x^{\prime}}\right]+\left[-p_{x^{\prime}}, \overline{x^{\prime}} p_{x^{\prime}}\right], \\
& 0=\left[\overline{x^{\prime}} p_{x^{\prime}}, \overline{y^{\prime}} p_{y^{\prime}}\right]+\left[\overline{y^{\prime}} p_{y^{\prime}},-p_{y^{\prime}}\right]+\left[-p_{y^{\prime}}, \overline{x^{\prime}} p_{x^{\prime}}\right], \\
& 0=\left[\overline{x^{\prime}} p_{x^{\prime}},-p_{x^{\prime}}\right]+\left[-p_{x^{\prime}},-p_{y^{\prime}}\right]+\left[-p_{y^{\prime}}, \overline{x^{\prime}} p_{x^{\prime}}\right] .
\end{aligned}
$$

By Eq. (18), all the six commutators (up to a sign) that appear in Eq. (19) are zero.

For a large enough $m$, after a possible redefinition of the unit cell, the movers $p_{x^{\prime}}, p_{y^{\prime}}$ will be supported only on two unit cells, one at the origin and another at $(1,0)$ or $(0,1)$. Thus, there are only six $=\left(\begin{array}{l}4 \\ 2\end{array}\right)$ potentially nontrivial commutators among all the translates (with respect to $R^{\prime}$ ) of $p_{x^{\prime}}$ and $p_{y^{\prime}}$, but we have made these six commutators to vanish.

Lemma III.5. For any nontrivial topological charge e, there exists a free basis for the stabilizer module (the columns of $\sigma$ ) such that e is represented by one basis element whose all components belong to the ideal $\mathfrak{m}=(x-1, y-1) \subset R$.

Proof. Let $\sigma$ be chosen such that $\epsilon=\sigma^{\dagger} \lambda_{q}$ satisfies Eq. (7). This is possible by [23, $\S 7$ Thm. 4]. Apply row operations $\operatorname{GL}\left(q ; \mathbb{F}_{p}\right)$ to $\epsilon$ so that $\left.\epsilon\right|_{x=1, y=1}$ is in the reduced row echelon form. The set of all topological charges is coker $\epsilon$ which is in fact a vector space over $\mathbb{F}_{p}$ on which the translation group has trivial action. The dimension $k=\operatorname{dim}_{\mathbb{F}_{p}} \operatorname{coker} \epsilon=\left.\operatorname{dim}_{\mathbb{F}_{p}} \operatorname{coker} \epsilon\right|_{x=1, y=1}$ is precisely the number of all zero rows of $\left.\epsilon\right|_{x=1, y=1}$. Hence, any nonzero element of the codomain of $\epsilon$ that is supported on these last $k$ components represents a nontrivial topological charge. Therefore, for any nontrivial charge $e$, its representative vector in coker $\epsilon$ can be mapped to a unit vector by some $\mathrm{GL}\left(k ; \mathbb{F}_{p}\right)$ acting on the last $k$ components.

Lemma III.6. Under a choice of a sufficiently large unit cell, the movers $p_{x}, p_{y}$ for a nontrivial boson e can be mapped to

$$
\begin{aligned}
& p_{x}=\left(\begin{array}{llllll}
1 & 0 & 0 & 0 & \cdots & 0
\end{array}\right)^{T}, \\
& p_{y}=\left(\begin{array}{llllll}
0 & 1 & 0 & 0 & \cdots & 0
\end{array}\right)^{T}
\end{aligned}
$$

by some elementary symplectic transformation $\operatorname{ESp}^{\dagger}(q ; R)$ (Clifford circuit).

Proof. By Lemma III.4, we may assume that $p_{x}$ and $p_{y}$ are supported on at most two adjacent unit cells. In particular, $p_{x}$ is a Laurent polynomial vector over $\mathbb{F}_{p}\left[x^{ \pm}\right]$, satisfying $p_{x}^{\dagger} \lambda_{q} p_{x}=0$. Since $\mathbb{F}_{p}\left[x^{ \pm}\right]$is a principal ideal domain, we can find an elementary symplectic transformation 
$E_{1} \in \operatorname{ESp}^{\dagger}\left(q ; \mathbb{F}_{p}\left[x^{ \pm}\right]\right)$that turns $p_{x}$ into a vector with a single nonzero component, say, $g$ at the first entry; see computation in $[23, \S 6]$. But the single component $g$ must be a monomial; under the choice of $\epsilon$ of Lemma III.5 we have $f g=x-1$ for some $f \in \mathfrak{m}=(x-1, y-1)$, and $x-1$ being irreducible in a unique factorization domain $R$ forces $g$ to be a unit.

After the transformation $E_{1}$, the $y$-mover becomes $E_{1} p_{y}=h_{1} y+h_{0}$ for some $h_{1}, h_{0} \in \mathbb{F}_{p}\left[x^{ \pm}\right]^{2 q}$. To remove the appearance of $x$ in this $y$-mover, we further take a coarse-graining $\chi_{\#}^{(m)}$ induced by a ring injection $\chi^{(m)}: x^{\prime} \mapsto x^{m}, y^{\prime} \mapsto y$. That is, $\chi_{\#}^{(m)}$ is a coarse-graining along $x$-direction only.

Assuming $m$ is even, let us fix a specific basis of $R \cong R^{\prime m}$ as an $R^{\prime}$-module: $R=x^{-m / 2} R^{\prime} \oplus$ $\cdots \oplus x^{m / 2-1} R^{\prime}$. This choice is convenient because any Laurent polynomial vector $v \in R^{2 q}$ becomes, by $\chi_{\#}^{(m)}$, a vector with entries in $\mathbb{F}_{p}[y]$ without $x$ for all sufficiently large $m$.

Once $x$-mover is put in the promised form, any further coarse-graining $\chi_{\#}^{(m)}$ does not complicate the $x$-mover; if $p_{x}$ is supported on a single cell, i.e., $p_{x}$ involves no variable, the operator $p_{x^{\prime}}=$ $\phi_{\#}^{(m)}\left(\left(x^{-m / 2}+x^{-m / 2+1}+\cdots+x^{m / 2-1}\right) p_{x}\right)$ is still supported on one new unit cell (no variables), and $\mathrm{ESp}^{\dagger}\left(m q ; \mathbb{F}_{p}\right)$, i.e., some Clifford transformation within a unit cell, can bring $p_{x^{\prime}}$ into the promised form. This transformation of $\operatorname{ESp}^{\dagger}\left(m q ; \mathbb{F}_{p}\right)$ is innocuous in that it does not insert or remove any variable to the $x$ - and $y$-movers. A benefit of $\chi_{\#}^{(m)}$ is now that $p_{y^{\prime}}=\chi_{\#}^{(m)}\left(E_{1} p_{y}\right)$ involves no $x^{\prime}$ and furthermore $p_{y^{\prime}}=t_{1} y+t_{0}$ for some $t_{1}, t_{0} \in \mathbb{F}_{p}^{2 m q}$.

Therefore, we may assume that $p_{x}$ is in the promised form with the sole nonzero entry 1 in the first component, and $p_{y}$ is a polynomial in $y$ with all exponents being 0 or 1 not involving $x$. Since $p_{x}^{\dagger} \lambda_{q} p_{y}=0$, the $q+1$-st component of $p_{y}$ must be zero. This forces $q \geq 2$. Now, we look at the $2,3, \ldots, q, q+2, q+3, \ldots, 2 q$-th components of $p_{y}$. If they generate the unit ideal $\left(=\mathbb{F}_{p}\left[y^{ \pm}\right]\right)$, then clearly $p_{y}$ can be turned into the promised form. If not, then by the exponent restriction we can use some transformation of $\operatorname{ESp}^{\dagger}\left(q-1 ; \mathbb{F}_{p}\right)$ to turn $p_{y}$ into one that has $y-v$ at the second component with $v \in \mathbb{F}_{p} \backslash\{0\}$, some $u \in \mathbb{F}_{p}$ at the first component, and zeros in all the other components. Then, under the choice of $\epsilon$ of Lemma III.5 we would have $(x-1) u+f(y-v)=y-1$ for some $f \in \mathfrak{m}$, but $u x-u-y+1=-f(y-v)$ is an irreducible polynomial, a contradiction.

Proof of Theorem II.2. Let $k$ be the $\mathbb{F}_{p}$-dimension of the torsion submodule of coker $\epsilon$. If $k=0$, then by [23, Lem. 7.1] $\sigma$ can be chosen to be kernel free, and coker $\epsilon$ must be pure torsion, which implies coker $\epsilon=0$, so the first Fitting ideal of $\epsilon$ is unit, and [23, Cor. 4.2] says that the code (ground space) on any finite periodic lattice encodes zero logical qudit. Then, [22, Thm. IV.4] provides a Clifford QCA that maps the stabilizer group to the trivial one. The result of [18] implies that this Clifford QCA is actually a Clifford circuit that is translation invariant with respect to a translation 
subgroup of a finite index.

If $k \neq 0$, as remarked earlier, we can assume Eq. (7). By Lemma III.3 we have a nontrivial boson, and its movers can be chosen as in Lemma III.6 by reducing the translation invariance to a subgroup of a finite index. Since these simplified movers have only one nonzero entry of 1 , under the choice of stabilizer generators in Lemma III.5 we must have

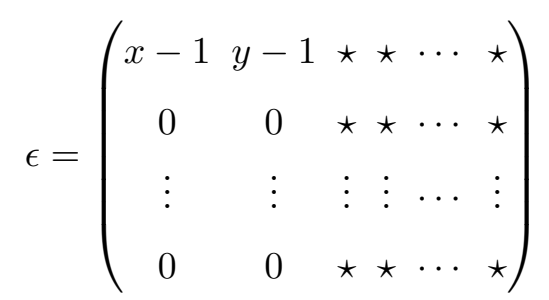

where $\star$ indicates an unknown entry, but the first row has entries from $\mathfrak{m}=(x-1, y-1)$. Since $\left(\begin{array}{lllll}y-1 & -x+1 & 0 & \cdots & 0\end{array}\right)^{T}$ is in $\operatorname{ker} \epsilon$, the condition $\operatorname{ker} \epsilon=\operatorname{im} \lambda_{q} \epsilon^{\dagger}$ implies that the rows of $\epsilon$ must generate

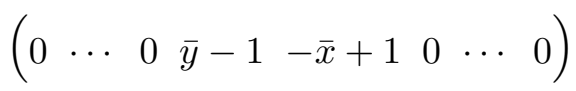

over $R$ where $\bar{y}-1$ is at the $q+1$-th position from the left. But the $R$-linear combination that results in this row vector cannot contain a nonzero summand of the first row in Eq. (21) because the base ring $R$ does not have any zero divisor. This makes it impossible for $k$ to be 1 since $k$ is the number of rows of $\epsilon$ that becomes zero by setting $x=1=y$; see the proof of Lemma III.5.

Let us extend $\epsilon$ to include a row Eq. (22) to make $\epsilon^{\prime}$; this amounts to increasing the number of generators for the stabilizer module by 1 . (The new $\epsilon^{\prime}$ does not satisfy Eq. (7) since coker $\epsilon^{\prime}$ is not torsion.)

$$
\epsilon^{\prime}=\left(\begin{array}{cccccccccc}
x-1 & y-1 & \star & \cdots & \star & u^{\prime} & v^{\prime} & \star & \cdots & \star \\
0 & 0 & 0 & \cdots & 0 & \bar{y}-1 & -\bar{x}+1 & 0 & \cdots & 0 \\
0 & 0 & \star & \cdots & \star & \star & \star & \star & \cdots & \star \\
\vdots & \vdots & \vdots & \cdots & \vdots & \vdots & \vdots & \vdots & \cdots & \vdots \\
0 & 0 & \star & \cdots & \star & \star & \star & \star & \cdots & \star
\end{array}\right)
$$

where $u^{\prime}, v^{\prime}$ are unknown. Since the first row of $\epsilon^{\prime}$ has entries in $\mathfrak{m}$, the two generators of $\mathfrak{m}$ in the top left can eliminate all $\star$ in the first row by control-Not and Hadamard gates which act on the 
right of $\epsilon^{\prime}$. The second row also remains intact. Thus we obtain

$$
\epsilon^{\prime \prime}=\left(\begin{array}{cccccccccc}
x-1 & y-1 & 0 & \cdots & 0 & u^{\prime \prime} & v^{\prime \prime} & 0 & \cdots & 0 \\
0 & 0 & 0 & \cdots & 0 & \bar{y}-1 & -\bar{x}+1 & 0 & \cdots & 0 \\
0 & 0 & \star & \cdots & \star & \star & \star & \star & \cdots & \star \\
\vdots & \vdots & \vdots & \cdots & \vdots & \vdots & \vdots & \vdots & \cdots & \vdots \\
0 & 0 & \star & \cdots & \star & \star & \star & \star & \cdots & \star
\end{array}\right) .
$$

Here, each pair of entries in the columns of $u^{\prime \prime}, v^{\prime \prime}$, below the second row, must be a $R$-multiple of $(\bar{y}-1-\bar{x}+1)$ because $\epsilon^{\prime \prime \dagger} \lambda_{q} \epsilon^{\prime \prime}=0$; the symplectic product with the first row enforces this. Hence, they can be eliminated by $\mathrm{GL}(q+1 ; R)$ on the left of $\epsilon^{\prime}$. Now we use the following elementary fact which will be proved shortly.

Lemma III.7. For $u, v \in R$, if a matrix

$$
M=\left(\begin{array}{cccc}
x-1 & y-1 & u & v \\
0 & 0 & \bar{y}-1 & -\bar{x}+1
\end{array}\right)
$$

satisfies $M \lambda_{2} M^{\dagger}=0$, then there exist $A \in \mathrm{GL}(2 ; R)$ and $B \in \mathrm{ESp}^{\dagger}(2 ; R)$ such that $A M B=\epsilon_{0}$ where $\epsilon_{0}$ is defined in Eq. (3).

Then we obtain

$$
\epsilon^{\prime \prime \prime}=\left(\begin{array}{cccccccccc}
x-1 & y-1 & 0 & \cdots & 0 & 0 & 0 & 0 & \cdots & 0 \\
0 & 0 & 0 & \cdots & 0 & \bar{y}-1 & -\bar{x}+1 & 0 & \cdots & 0 \\
0 & 0 & \star & \cdots & \star & 0 & 0 & \star & \cdots & \star \\
\vdots & \vdots & \vdots & \cdots & \vdots & \vdots & \vdots & \vdots & \cdots & \vdots \\
0 & 0 & \star & \cdots & \star & 0 & 0 & \star & \cdots & \star
\end{array}\right) .
$$

It is evident that $\epsilon_{0}$ is a direct summand. The change $\epsilon \rightarrow \epsilon^{\prime} \rightarrow \epsilon^{\prime \prime} \rightarrow \epsilon^{\prime \prime \prime}$ is achieved by $\operatorname{ESp}^{\dagger}(q ; R)$ and change of a generating set of the stabilizer module. The complement of $\epsilon_{0}$ in $\epsilon^{\prime \prime \prime}$ gives a stabilizer module that satisfies the conditions of our theorem. This proves the induction step decreasing $k$ by 2 , and thus completes the proof of the theorem.

Proof of Lemma III. \%. By long division we can write $u=(\bar{y}-1) u^{\prime}+u^{\prime \prime}$ where $u^{\prime} \in R, u^{\prime \prime} \in \mathbb{F}_{p}\left[x^{ \pm}\right]$. By a row operation on the left of $M$, we can eliminate $(\bar{y}-1) u^{\prime}$ and thus we assume from now on that $u=u^{\prime \prime} \in \mathbb{F}_{p}\left[x^{ \pm}\right]$. The equation $M \lambda_{2} M^{\dagger}=0$ implies that $(x-1) \bar{u}+(y-1) \bar{v}=(\bar{x}-1) u+(\bar{y}-1) v$, which can be rearranged as

$$
(x-1)(\bar{u}+\bar{x} u)=-(y-1)(\bar{v}+\bar{y} v) .
$$


The left hand side is a Laurent polynomial in $x$, and therefore, if nonzero, it is not divisible by $y-1$. Hence, $\bar{u}+\bar{x} u=0=\bar{v}+\bar{y} v$. Let $u=\sum_{j} u_{j} x^{j}$ where $u_{j} \in \mathbb{F}_{p}$ be the expansion of $u$. It follows that $u_{-j}+u_{j+1}=0$ for all $j$. This implies that $\left.u\right|_{x=1}=0$ so $u=(x-1) h$ for some $h \in \mathbb{F}_{p}\left[x^{ \pm}\right]$. Substituting, we have $\bar{u}+\bar{x} u=(\bar{x}-1) \bar{h}-(\bar{x}-1) h=0$ or $h=\bar{h}$. Thus the control-Phase gate on the first qudit can eliminate $u$.

We are left with an equation $\bar{v}+\bar{y} v=0$. Write $v=(x-1) s+f$ where $f \in \mathbb{F}_{p}\left[y^{ \pm}\right]$. Then, we have $(x-1)(-\bar{x} \bar{s}+\bar{y} s)+\bar{f}+\bar{y} f=0$. Since $f$ is constant in $x$, we have $f+\bar{y} f=0$ implying, as before, that $f=(y-1) g$ for some $g=\bar{g} \in \mathbb{F}_{p}\left[y^{ \pm}\right]$, and hence $f$ can be eliminated by the control-Phase on the second qudit.

The remaining term $(x-1) s$ of $v$ satisfies $\bar{x} y \bar{s}=s$. We claim that $s=y \bar{r}+\bar{x} r$ for some $r \in R$, which will conclude the proof:

$$
\left(\begin{array}{cc}
1 & -r \\
0 & 1
\end{array}\right)\left(\begin{array}{cccc}
x-1 & y-1 & 0 & (x-1) s \\
0 & 0 & \bar{y}-1 & -\bar{x}+1
\end{array}\right)\left(\begin{array}{cccc}
1 & 0 & 0 & -y \bar{r} \\
0 & 1 & -\bar{y} r & 0 \\
0 & 0 & 1 & 0 \\
0 & 0 & 0 & 1
\end{array}\right)=\left(\begin{array}{cccc}
x-1 & y-1 & 0 & 0 \\
0 & 0 & \bar{y}-1 & -\bar{x}+1
\end{array}\right) .
$$

To show the claim, let $z=\bar{x} y$. Write $s=\sum_{j} y^{j} s_{j}$ where $s_{j} \in \mathbb{F}_{p}\left[z^{ \pm}\right]$; such an expression is unique as seen by considering a ring isomorphism $\mathbb{F}_{p}\left[z^{ \pm}, y^{ \pm}\right] \rightarrow \mathbb{F}_{p}\left[x^{ \pm}, y^{ \pm}\right]$where $z \mapsto \bar{x} y, y \mapsto y$. We have $s_{-j}=z \bar{s}_{j}$ for all $j$ and hence $s_{0}=(z+1) \ell$ for some $\ell=\bar{\ell} \in \mathbb{F}_{p}\left[z^{ \pm}\right]$. Let $s_{+}=\sum_{j>0} y^{j} s_{j}$. Then $s=s_{+}+(z+1) \ell+z \overline{s_{+}}=y \overline{\left(x \ell+x s_{+}\right)}+\bar{x}\left(x \ell+x s_{+}\right)$.

\section{ACKNOWLEDGMENTS}

I thank Lukasz Fidkowski and Matt Hastings for fruitful discussions and Zhenghan Wang for informing me of [13]. I also thank an anonymous referee for comments that helped improving the clarity of the manuscript.

[1] Robbert Dijkgraaf and Edward Witten, "Topological gauge theories and group cohomology," Communications in Mathematical Physics 129, 393-429 (1990).

[2] Michael A. Levin and Xiao-Gang Wen, "String-net condensation: a physical mechanism for topological phases," Physical Review B 71, 045110 (2005), arXiv:cond-mat/0404617. 
[3] Kevin Walker and Zhenghan Wang, "(3+1)-TQFTs and topological insulators," Frontiers of Physics 7, 150-159 (2011), arXiv:1104.2632.

[4] A. R. Calderbank and Peter W. Shor, "Good quantum error-correcting codes exist," Phys. Rev. A 54, 1098-1105 (1996), quant-ph/9512032.

[5] Andrew Steane, "Multiple particle interference and quantum error correction," Proc. Roy. Soc. Lond. A 452, 2551 (1996), quant-ph/9601029.

[6] Daniel Gottesman, "A class of quantum error-correcting codes saturating the quantum hamming bound," Phys. Rev. A 54, 1862 (1996), quant-ph/9604038.

[7] A. R. Calderbank, E. M Rains, P. W. Shor, and N. J. A. Sloane, "Quantum error correction and orthogonal geometry," Phys. Rev. Lett. 78, 405-408 (1997), quant-ph/9605005.

[8] Xiao-Gang Wen, "Mean-field theory of spin-liquid states with finite energy gap and topological orders," Phys. Rev. B 44, 2664-2672 (1991).

[9] A.Yu. Kitaev, "Fault-tolerant quantum computation by anyons," Annals of Physics 303, 2-30 (2003), arXiv:quant-ph/9707021.

[10] Sagar Vijay, Jeongwan Haah, and Liang Fu, "Fracton topological order, generalized lattice gauge theory and duality," Phys. Rev. B 94, 235157 (2016), arXiv:1603.04442.

[11] Bojko Bakalov and Alexander Kirillov Jr., Lectures on Tensor Categories and Modular Functors, University Lecture Series, Vol. 21 (American Mathematical Society, 2001).

[12] A. Kitaev, "Anyons in an exactly solved model and beyond," Annals of Physics 321, 2-111 (2006), arXiv:cond-mat/0506438.

[13] César Galindo and Nicolás Jaramillo Torres, "Solutions of the hexagon equation for abelian anyons," arXiv:1606.01414v3.

[14] T. Y. Lam, Introduction to Quadratic Forms over Fields, Graduate studies in mathematics 67 (American Mathematical Society, 2004).

[15] Richard Elman, Nikita Karpenko, and Alexander Merkurjev, The Algebraic and Geometric Theory of Quadratic Forms, Colloquium Publications, Vol. 56 (American Mathematical Society, 2008).

[16] Héctor Bombín, "Structure of 2D topological stabilizer codes," Commun. Math. Phys. 327, 387-432 (2014), arXiv:1107.2707.

[17] M. Freedman and Matthew B Hastings, "Classification of quantum cellular automata," (2019), arXiv:1902.10285.

[18] Jeongwan Haah, "Clifford quantum cellular automata: Trivial group in 2d and Witt group in 3d," (2019), arXiv:1907.02075.

[19] Dirk-M. Schlingemann, Holger Vogts, and Reinhard F. Werner, "On the structure of Clifford quantum cellular automata," Journal of Mathematical Physics 49, 112104 (2008), arXiv:0804.4447.

[20] F. Gabbiani J. Fröhlich, "Braid statistics in local quantum theory," Rev. Math. Phys. 2, 251-353 (1990).

[21] Jeongwan Haah, "Algebraic methods for quantum codes on lattices," Revista Colombiana de Matemáticas 50, 295-345 (2016), arXiv:1607.01387. 
[22] Jeongwan Haah, Lukasz Fidkowski, and Matthew B. Hastings, "Nontrivial quantum cellular automata in higher dimensions," arXiv:1812.01625.

[23] Jeongwan Haah, "Commuting Pauli hamiltonians as maps between free modules," Commun. Math. Phys. 324, 351-399 (2013), arXiv:1204.1063.

[24] Sergey Bravyi, Matthew Hastings, and Spyridon Michalakis, "Topological quantum order: Stability under local perturbations," J. Math. Phys. 51, 093512 (2010), arXiv:1001.0344.

[25] Michael Pretko, "Subdimensional particle structure of higher rank U(1) spin liquids," Phys. Rev. B 95, 115139 (2017), arXiv:1604.05329.

[26] A. M. Polyakov, "Compact gauge fields and the infrared catestrophe," Physics Letters B 59, 82-84 (1975).

[27] M. B. Hastings, "Classifying quantum phases with the torus trick," Phys. Rev. B 88, 165114 (2013), arXiv:1305.6625.

[28] Xiao-Gang Wen, "Quantum orders in an exact soluble model," Phys. Rev. Lett. 90, 016803 (2003), arXiv:quant-ph/0205004.

[29] Michael Levin and Xiao-Gang Wen, "Fermions, strings, and gauge fields in lattice spin models," Phys. Rev. B 67, 245316 (2003), arXiv:cond-mat/0302460. 